\title{
Search for continuous gravitational waves from Fomalhaut $b$ in the second Advanced LIGO observing run with a hidden Markov model
}

\author{
Dana Jones $\oplus^{1, *}$ and Ling Sun $\circledast^{2,3, \dagger}$ \\ ${ }^{1}$ College of Arts and Sciences, University of Pennsylvania, Philadelphia, Pennsylvania 19104, USA \\ ${ }^{2}$ LIGO Laboratory, California Institute of Technology, Pasadena, California 91125, USA \\ ${ }^{3}$ OzGrav-ANU, Centre for Gravitational Astrophysics, College of Science, \\ The Australian National University, Australian Capital Territory 2601, Australia
}

(Received 16 July 2020; accepted 24 December 2020; published 21 January 2021)

\begin{abstract}
Results are presented from a semicoherent search for continuous gravitational waves from a nearby neutron star candidate, Fomalhaut b, using data collected in the second observing run of Advanced LIGO. The search is based on a hidden Markov model scheme, capable of tracking signal frequency evolution from the star's secular spin-down and stochastic timing noise simultaneously. The scheme is combined with a frequency domain matched filter $(\mathcal{F}$-statistic), calculated coherently over five-day time stretches. The frequency band $100-1000 \mathrm{~Hz}$ is searched. After passing the above-threshold candidates through a hierarchy of vetoes, one candidate slightly above the $1 \%$ false alarm probability threshold remains for further scrutiny. No strong evidence of continuous waves is found. We present the strain upper limits in the full frequency band searched at $90 \%$ confidence level.
\end{abstract}

DOI: 10.1103/PhysRevD.103.023020

\section{INTRODUCTION}

Gravitational waves (GWs), perturbations in spacetime that propagate at the speed of light, were first directly observed in 2015 when the Advanced Laser Interferometer Gravitational-Wave Observatory (Advanced LIGO) detected a merging binary black hole system (GW150914) [1,2]. The Virgo detector joined the observation at the end of the second observing run (O2) in 2017 [3]. In the years since the first detection, the sensitivity of these interferometers has been greatly improved, allowing for increasingly frequent detections of compact binary coalescences (CBCs) [4-7]. Other types of GW sources that also radiate at frequencies within the observational band of ground-based interferometers remain yet undetected, e.g., the persistent, well modeled, continuous gravitational waves (CWs) produced by isolated spinning neutron stars. These CWs, if detected, will provide invaluable information regarding the structure of the neutron stars as well as the nuclear equation of state in extremepressure situations [8]. A great deal of work has been carried out to develop methods and conduct searches for CWs. There are three main types of CW searches: (1) targeted searches for pulsars whose sky positions and ephemerides are well measured electromagnetically (e.g., $[9,10]$ ), (2) directed searches for neutron stars with known sky positions but unknown rotation frequencies (e.g., [11-17]),

\footnotetext{
*dhjones@sas.upenn.edu

†ling.sun@anu.edu.au
}

and (3) all-sky searches, surveys done over the whole sky to search for emitting sources (e.g., [18]).

In this paper, a directed search, more expensive than a targeted search but less expensive than an all-sky search, is conducted in the Advanced LIGO O2 data [19] for a nearby neutron start candidate, Fomalhaut b. Fomalhaut b was originally hypothesized to be an exoplanet orbiting around the star Fomalhaut A [20,21]. However, certain peculiarities, namely its nondetection in the infrared and its potentially highly eccentric orbit, have led to speculation over whether it is in fact an exoplanet. A different hypothesis has been gaining momentum; rather than a companion object, Fomalhaut b may in fact be a background neutron star to Fomalhaut A [22]. There has also been recent evidence that Fomalhaut b may be a directly imaged catastrophic collision between two large planetesimals in an extrasolar planetary system [23]. Although the remaining uncertainty regarding the source's identity may make it a less promising candidate for a CW search, Fomalhaut b remains of interest due to its extremely close proximity. It is currently hypothesized to be just 11 pc away, which would make it, assuming the neutron star hypothesis to be true, the closest known neutron star to our solar system [22]. A CW search directed at Fomalhaut b was conducted in the first observing run of Advanced LIGO, but no evidence of gravitational radiation was found [13].

Since the expected strain amplitudes of CWs are orders of magnitude smaller than those produced by CBCs, vast computational resources are required to integrate observational data coherently over a long period of time (e.g., $\sim 1 \mathrm{yr}$ ), searching for the signal frequency and the time 
derivatives [8]. In addition, intrinsic, stochastic frequency wandering, or "timing noise," associated with the secular spin-down of a star could degrade the sensitivity of a fully coherent search [24]. Thus, although fully coherent searches are still of interest in certain systems with negligible timing noise, we conduct a computationally efficient semicoherent search based on a hidden Markov model (HMM) scheme, equipped to track the evolving signal frequency due to secular spin-down and stochastic timing noise [25]. The tracking scheme has its origins in engineering and has recently been used in many CW searches (e.g., [12,15,26-29]). In this search, we assume that the signal frequency evolution is dominated by the star's secular spin-down, allowing for minor stochastic timing noise, as the star is an isolated source (cf. the signal evolution is expected to be dominated by timing noise if the source is in an accreting binary system).

The search presented in this paper is conducted using the Advanced LIGO O2 data collected from January 3 to August 25, 2017, in the frequency band $100-1000 \mathrm{~Hz}$, divided into $1 \mathrm{~Hz}$ subbands to parallelize the computation. The total observing duration is split into five-day coherent segments in order to balance search sensitivity and computing cost in the presence of timing noise. The short Fourier transforms (SFTs) of the data are passed through a frequency domain matched filter (the $\mathcal{F}$-statistic). These coherent five-day segments are combined incoherently using a HMM tracking scheme. The search output is then passed through a hierarchy of veto validations. No strong evidence of continuous waves is found.

The organization of the paper is as follows. Section II outlines the methods used in the search. Section III details the search setup, including source parameters, search configuration, threshold, and sensitivity estimates. In Sec. IV, we explain the results from the search, including the five vetoes applied to the overthreshold candidates, and discuss future work that could be used to further follow up on the results. We also present the upper limits obtained on the signal strain and source properties. Finally, the conclusion is given in Sec. V.

\section{METHODS}

This search is composed of two main procedures: (1) coherently summing up the signal power over consecutive five-day time stretches using the $\mathcal{F}$-statistic, and (2) a HMM tracking to find the most probable signal evolution path over the total observing run. The signal model is briefly reviewed in Sec. II A. The two procedures are described in Secs. II B and II C, respectively. The detection statistic adopted in this search, the Viterbi score, is defined in Sec. II D.

\section{A. Signal model}

The phase of the signal as observed in the detector can be described as [30]

$$
\Phi(t)=2 \pi \sum_{k=0}^{s} \frac{f_{0}^{(k)} t^{k+1}}{(k+1) !}+\frac{2 \pi}{c} \hat{n} \cdot \vec{r}(t) \sum_{k=0}^{s} \frac{f_{0}^{(k)} t^{k}}{k !},
$$

where $f_{0}$ is the signal frequency at reference time $t=0$, the superscript $(k)$ denotes the $k$ th time derivative of the signal frequency, $\hat{n}$ is the unit vector directed outward from the solar system barycenter (SSB) to the neutron star, and $\vec{r}(t)$ is the position vector of the detector relative to the SSB. Then, the signal can be expressed as

$$
h(t)=\mathcal{A}^{\mu} h_{\mu}(t),
$$

where $\mathcal{A}^{\mu}$, depending on the characteristic gravitationalwave strain amplitude $h_{0}$, source orientation, and signal initial phase, represents the amplitudes associated with the four linearly independent components [30]

$$
\begin{aligned}
& h_{1}(t)=a(t) \cos \Phi(t), \\
& h_{2}(t)=b(t) \cos \Phi(t), \\
& h_{3}(t)=a(t) \sin \Phi(t), \\
& h_{4}(t)=b(t) \sin \Phi(t) .
\end{aligned}
$$

In (3)-(6), $a(t)$ and $b(t)$ are the antenna-pattern functions given by Eqs. (12) and (13) in Ref. [30], and $\Phi(t)$ is the signal phase in (1).

\section{B. $\mathcal{F}$-statistic}

The $\mathcal{F}$-statistic is a matched filter used to estimate the likelihood that a signal described above is present in the frequency domain. The time-domain data $x(t)$ collected by the detector can be written as

$$
x(t)=\mathcal{A}^{\mu} h_{\mu}(t)+n(t),
$$

where $n(t)$ is stationary, additive noise [30]. First, a scalar product as a sum over single-detector inner products is defined as

$$
\begin{aligned}
(x \mid y) & =\sum_{X}\left(x^{X} \mid y^{X}\right) \\
& =\sum_{X} 4 \Re \int_{0}^{\infty} d f \frac{\tilde{x}^{X}(f) \tilde{y}^{X *}(f)}{S_{h}^{X}(f)},
\end{aligned}
$$

where $X$ indexes the detector, $S_{h}^{X}(f)$ is the single-sided power spectral density (PSD) of detector $X$, the tilde denotes a Fourier transform, and $\Re$ is the real part of a complex number [31]. Then, the $\mathcal{F}$-statistic can be written in the form

$$
\mathcal{F}=\frac{1}{2} x_{\mu} \mathcal{M}^{\mu \nu} x_{\nu}
$$

where $x_{\mu}=\left(x \mid h_{\mu}\right)$ and $\mathcal{M}^{\mu \nu}$ represents the matrix inverse of $\mathcal{M}_{\mu \nu}=\left(h_{\mu} \mid h_{\nu}\right)$ [32]. If the noise is Gaussian and the 
single-sided PSD is the same in all detectors, the probability of having a signal in the data solely depends on the signal-to-noise ratio, given by [30]

$$
\rho_{0}^{2}=\frac{K h_{0}^{2} T_{\text {coh }}}{S_{h}(f)}
$$

where $K$ is a constant that depends on the sky location, orientation of the source, and number of detectors, and $T_{\text {coh }}$ is the length of data combined coherently.

\section{Hidden Markov model}

This semicoherent search, based on the HMM scheme, employs the Viterbi algorithm to identify the most likely frequency evolution path of the signal. (See Ref. [33] for an explanation of the classic Viterbi algorithm.) It is computationally efficient and robust in the presence of timing noise [25].

A Markov chain is a stochastic process that transitions from one discrete state to another at discrete times. A hidden Markov model is composed of two variables: the unobservable, hidden state variable $q(t) \in\left\{q_{1}, \ldots, q_{N_{Q}}\right\}$ and the observable, measurement state variable $o(t) \in$ $\left\{o_{1}, \ldots, o_{N_{O}}\right\}$, where $N_{Q}$ and $N_{O}$ are the total number of hidden and measurement states, respectively. For any time $t_{n+1}$, the hidden state is solely dependent on the state at time $t_{n}$ and has a transition probability of

$$
A_{q_{j} q_{i}}=\operatorname{Pr}\left[q\left(t_{n+1}\right)=q_{j} \mid q\left(t_{n}\right)=q_{i}\right] .
$$

The hidden state $q_{i}$, present in the observable state $o_{j}$, has an emission probability defined as

$$
L_{o_{j} q_{i}}=\operatorname{Pr}\left[o\left(t_{n}\right)=o_{j} \mid q\left(t_{n}\right)=q_{i}\right]
$$

at time $t_{n}$. The prior is written as

$$
\Pi_{q_{i}}=\operatorname{Pr}\left[q\left(t_{1}\right)=q_{i}\right],
$$

where $t_{1}$ is the reference time of the first time step. The probability that an observed sequence $O=o\left(t_{1}\right), \ldots, o\left(t_{N_{T}}\right)$, where $N_{T}$ is the total number of time steps, is the result of a hidden state path $Q=q\left(t_{1}\right), \ldots, q\left(t_{N_{T}}\right)$ via a Markov chain can be described by

$$
\begin{aligned}
\operatorname{Pr}(Q \mid O) \propto & L_{o\left(t_{N_{T}}\right) q\left(t_{N_{T}}\right)} A_{q\left(t_{N_{T}}\right) q\left(t_{N_{T}-1}\right)} \cdots L_{o\left(t_{2}\right) q\left(t_{2}\right)} \\
& \times A_{q\left(t_{2}\right) q\left(t_{1}\right)} \Pi_{q\left(t_{1}\right)} .
\end{aligned}
$$

The most probable path, calculated by maximizing $\operatorname{Pr}(Q \mid O)$, is [25]

$$
Q^{*}(O)=\arg \max \operatorname{Pr}(Q \mid O),
$$

where $\arg \max (\cdots)$ returns the argument that maximizes $(\cdots)$.

In this search, the one-dimensional state variable $q(t)$ is defined as $f_{0}(t)$. The discrete hidden states are mapped one-to-one to the frequency bins that make up the output of $\mathcal{F}(f)$ calculated over the span of length $T_{\text {coh }}$ (see Sec. II B). Each frequency bin size is then $\Delta f=1 /\left(2 T_{\text {coh }}\right)$. We choose $T_{\text {coh }}$ to satisfy

$$
\left|\int_{t}^{t+T_{\mathrm{coh}}} d t^{\prime} \dot{f_{0}}\left(t^{\prime}\right)\right|<\Delta f
$$

for $0<t<T_{\text {obs }}-T_{\text {coh }}$, where $T_{\text {obs }}$ is the total observation time. (See Ref. [25] for more details.)

Assuming that the frequency evolution caused by timing noise is much slower than that due to the star's secular spindown, and $\left|\dot{f}_{0}(t)\right|$ lies in the range between zero and the maximum estimated spin-down rate $\left|\dot{f}_{0}\right|_{\max }$, the signal frequency evolution can be approximated by a negatively biased random walk with $\left|\dot{f}_{0}(t)\right| \in\left[0,\left|\dot{f}_{0}\right|_{\max }\right]$. By substituting $\left|\dot{f}_{0}\right|_{\max }$ into (17), we can simplify (12) to become

$$
A_{q_{i-1} q_{i}}=A_{q_{i} q_{i}}=\frac{1}{2},
$$

with all other $A_{q_{j} q_{i}}$ entries vanishing. Then, using the definition of $\mathcal{F}$-statistic, the emission probability is defined as

$$
\begin{aligned}
L_{o(t) q_{i}} & =\operatorname{Pr}\left[o(t) \mid f_{i} \leq f_{0}(t) \leq f_{i}+\Delta f\right] \\
& \propto \exp \left[\mathcal{F}\left(f_{i}\right)\right]
\end{aligned}
$$

from $t$ to $t+T_{\text {coh }}$, where $f_{i}$ represents the central frequency in the $i$ th bin. A uniform prior of $\Pi_{q_{i}}=N_{Q}^{-1}$ is selected because there is no independent knowledge of $f_{0}$ [25].

The algorithm outputs the most likely frequency evolution path $Q^{*}(O)$ over the course of $T_{\mathrm{obs}}$. This is called the Viterbi path and consists of a frequency estimated at each discrete time step. Figure 1 shows an example of tracking a circularly polarized synthetic signal with $h_{0}=$ $1.57 \times 10^{-26}$ and $\cos \imath=1$, where $t$ is the source inclination angle, starting at $f_{0}=155.3 \mathrm{~Hz}$ (a frequency chosen randomly), and injected into Gaussian noise with amplitude spectral density (ASD) $S_{h}^{1 / 2}=4 \times 10^{-24} \mathrm{~Hz}^{-1 / 2}$. The total observing time tracked is 234 days, with $T_{\text {coh }}=5 \mathrm{~d}$. The first and second time derivatives of the injected signal frequency are $\dot{f_{0}}=-2 \times 10^{-12} \mathrm{~Hz} \mathrm{~s}^{-1}$ and $\ddot{f}_{0}=$ $2 \times 10^{-24} \mathrm{~Hz} \mathrm{~s}^{-2}$. The optimal signal evolution path reconstructed by the Viterbi algorithm (red dots) is plotted over the injected signal path (blue curve). The reconstructed path shows a stairstep pattern because the HMM method uses discrete frequency bins (shown as dashed lines). The injected signal, by contrast, evolves continuously. 


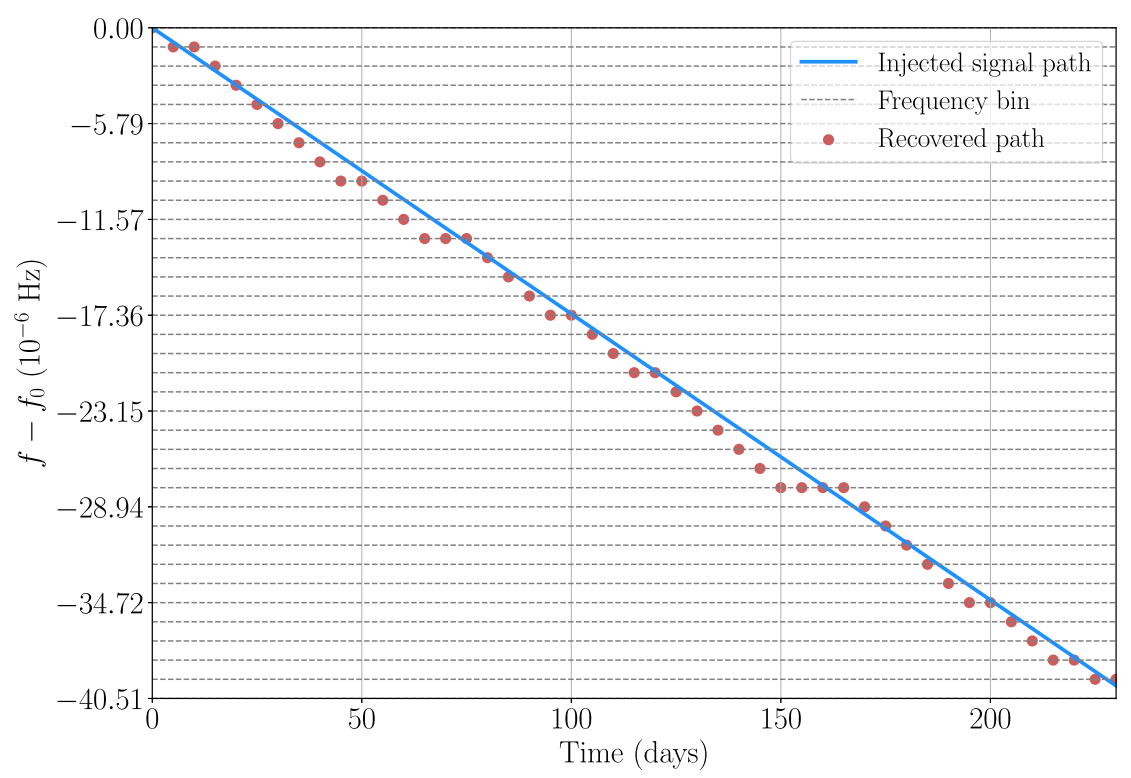

FIG. 1. HMM tracking of a synthetic signal with $h_{0}=1.57 \times 10^{-26}$ and $\cos \imath=1$, injected into Gaussian noise $\left(\mathrm{ASD} S_{h}^{1 / 2}=\right.$ $4 \times 10^{-24} \mathrm{~Hz}^{-1 / 2}$ ) at a starting frequency of $155.3 \mathrm{~Hz}$. The blue curve represents the injected signal (with $\dot{f_{0}}=-2 \times 10^{-12} \mathrm{~Hz} \mathrm{~s}^{-1}$ and $\ddot{f_{0}}=2 \times 10^{-24} \mathrm{~Hz} \mathrm{~s}^{-2}$ ), and the red dots represent the optimal Viterbi path recovered by the HMM tracking. The dashed lines indicate the size of the discrete frequency bins.

The recovered path matches the injected signal well in that it never strays more than two bins from the true frequency. The root-mean-square error between the two paths is $7.8 \times 10^{-7} \mathrm{~Hz}$, smaller than the discrete bin size, $1.16 \times 10^{-6} \mathrm{~Hz}$.

\section{Viterbi score}

We follow the existing literature and define the Viterbi score to evaluate the significance of any candidate output from the search $[25,26]$. In each subband searched (with width $1 \mathrm{~Hz}$ in this paper), the Viterbi score $S$ is defined such that the log likelihood of the optimal Viterbi path is equal to the mean log likelihood of all paths ending in different bins of the subband plus $S$ standard deviations at final step $N_{T}$. This is shown as follows:

$$
S=\frac{\ln \delta_{q^{*}}\left(t_{N_{T}}\right)-\mu_{\ln \delta}\left(t_{N_{T}}\right)}{\sigma_{\ln \delta}\left(t_{N_{T}}\right)},
$$

where

$$
\mu_{\ln \delta}\left(t_{N_{T}}\right)=N_{Q}^{-1} \sum_{i=1}^{N_{Q}} \ln \delta_{q_{i}}\left(t_{N_{T}}\right)
$$

and

$$
\sigma_{\ln \delta}\left(t_{N_{T}}\right)^{2}=N_{Q}^{-1} \sum_{i=1}^{N_{Q}}\left[\ln \delta_{q_{i}}\left(t_{N_{T}}\right)-\mu_{\ln \delta}\left(t_{N_{T}}\right)\right]^{2} .
$$

Here, $\delta_{q_{i}}\left(t_{N_{T}}\right)$ is the maximum probability of the path that ends in state $q_{i}\left(1 \leq i \leq N_{Q}\right)$ at step $N_{T}$, and $\delta_{q^{*}}\left(t_{N_{T}}\right)$ is the likelihood of the optimal Viterbi path (i.e., the probability $\operatorname{Pr}\left[Q^{*}(O) \mid O\right]$ ). The higher the score $S$, the more likely that a signal is present in the subband.

\section{SEARCH SETUP}

In this section, we first discuss the source parameters and the parameter space covered in the search in Sec. III A. Sections III B and III C describe the detection threshold and estimated search sensitivity, respectively.

\section{A. Source parameters and search configuration}

Fomalhaut b is located at right ascension $22 \mathrm{~h} 57 \mathrm{~m}$ $39.1 \mathrm{~s}$ and declination $29^{\circ} 37^{\prime} 20.0^{\prime \prime}$ (J2000). Reference [13] considered both the most optimistic and pessimistic estimates of Fomalhaut b's distance $(0.011-0.02 \mathrm{kpc})$ and age (316-3000 kyr) in the literature.

Here, we mainly rely on the estimated, age-based spindown rate in order to choose an optimal $T_{\text {coh }}$ in this search. The age-based $\dot{f_{0}}$ is estimated using $[13,25]$

$$
-\frac{f_{0}}{\left(n_{\min }-1\right) t_{\mathrm{age}}} \leq \dot{f_{0}} \leq-\frac{f_{0}}{\left(n_{\max }-1\right) t_{\mathrm{age}}},
$$

where $t_{\text {age }}$ is the age of the source and $n_{\min }$ and $n_{\max }$, respectively, represent the minimum and maximum values of the breaking index $n=f_{0} \ddot{f}_{0} / \dot{f}_{0}^{2}$. Given an estimated 
TABLE I. Estimated ranges of $\left|\dot{f_{0}}\right|$ and $T_{\text {coh }}$. The frequency range considered is $100-1000 \mathrm{~Hz}$.

\begin{tabular}{lccl}
\hline \hline$n$ & $t_{\text {age }}[\mathrm{kyr}]$ & $\left|\dot{f}_{0}\right| \mathrm{Hz} \mathrm{s}^{-1}$ & $T_{\text {coh }}[$ days $]$ \\
\hline 2 & 316 & {$\left[1.00 \times 10^{-11}, 1.00 \times 10^{-10}\right]$} & {$[0.82,2.58]$} \\
2 & 3000 & {$\left[1.06 \times 10^{-12}, 1.06 \times 10^{-11}\right]$} & {$[2.52,7.96]$} \\
5 & 316 & {$\left[2.51 \times 10^{-12}, 2.51 \times 10^{-11}\right]$} & {$[1.63,5.17]$} \\
5 & 3000 & {$\left[2.64 \times 10^{-13}, 2.64 \times 10^{-12}\right]$} & {$[5.03,15.92]$} \\
7 & 316 & {$\left[1.67 \times 10^{-12}, 1.67 \times 10^{-11}\right]$} & {$[2.00,6.33]$} \\
7 & 3000 & {$\left[1.76 \times 10^{-13}, 1.76 \times 10^{-12}\right]$} & {$[6.17,19.50]$} \\
\hline \hline
\end{tabular}

$\dot{f_{0}}$, a $T_{\text {coh }}$ value may then be chosen based on the following equation:

$$
T_{\text {coh }} \leq\left(2\left|\dot{f_{0}}\right|\right)^{-1 / 2},
$$

such that (17) is satisfied. Since the search sensitivity improves as $T_{\text {coh }}$ increases for a given $T_{\text {obs }}$ [25], we usually set $T_{\text {coh }}=\left(2\left|\dot{f_{0}}\right|\right)^{-1 / 2}$.

The frequency range searched in this analysis is 100 $1000 \mathrm{~Hz}$, where the Advanced LIGO detectors are most sensitive. Table I lists the $\left|\dot{f_{0}}\right|$ ranges calculated using (24), and the corresponding $T_{\text {coh }}$ ranges calculated using (25), for $f_{0} \in[100,1000] \mathrm{Hz}$. The ranges are calculated for each $n \in\{2,5,7\}$ and $t_{\text {age }} \in\{316,3000\}$ kyr. Since the uncertainty of the estimated $t_{\text {age }}$ is large, we select an intermediate $T_{\text {coh }}=5 \mathrm{~d}$, corresponding to a $\dot{f_{0}}$ range of $\dot{f_{0}} \in\left[-2.68 \times 10^{-12}, 0\right] \mathrm{Hz} \mathrm{s}^{-1}$. With this choice of $T_{\text {coh }}$, the search can cover most of the interesting parameter space, if the torque is dominated by gravitational radiation reaction $(n=5)$ or $r$-mode oscillations $(n=7)$, e.g., almost the full $100-1000 \mathrm{~Hz}$ band if $t_{\text {age }} \sim 10^{3} \mathrm{kyr}$, or the most sensitive hundred-hertz band if $t_{\text {age }} \sim 10^{2} \mathrm{kyr}$. Signals with $\dot{f_{0}}$ out of the covered range during part of the observing run could still be partially tracked by the HMM. The desired sensitivity of the search (Sec. III C), however, cannot be achieved. Note that although the minimum $T_{\text {coh }}=0.82 \mathrm{~d}$ in Table I could cover all scenarios listed above, it is not the optimal choice since the sensitivity degrades as $T_{\text {coh }}$ decreases $\left(\propto T_{\mathrm{coh}}^{-1 / 4}\right)$ [25].

The total observation period searched is the duration of Advanced LIGO's O2 run, excluding the first few weeks when the data quality was not optimal and was followed by a brief end-of-the-year break; that is, Global Positioning System (GPS) time 1167545066-1187733592 (January 3 to August 25, 2017), a total duration of about 234 days. Given the chosen $T_{\text {coh }}=5 \mathrm{~d}$, we have the total number of steps $N_{T}=47$.

\section{B. Threshold}

The Viterbi score threshold $S_{\text {th }}$, corresponding to a desired false alarm probability $\alpha_{\mathrm{f}}$ in each subband $\left(\alpha_{\mathrm{f}}=1 \%\right.$ in this search), is determined through Gaussian noise simulations.
In each of the five sample $1 \mathrm{~Hz}$ subbands, starting from $100 \mathrm{~Hz}, 300 \mathrm{~Hz}, 500 \mathrm{~Hz}, 700 \mathrm{~Hz}$, and $900 \mathrm{~Hz}, 200$ realizations of pure Gaussian noise are generated and searched with the same configuration as used in the real search (i.e., $T_{\text {coh }}=5 \mathrm{~d}$ and $T_{\text {obs }}=234 \mathrm{~d}$ ). The resulting 1000 scores are sorted and then the score at the 99th percentile is determined as the threshold, $S_{\text {th }}=6.95$. Ten out of the 1000 scores obtained from pure noise are above $S_{\text {th }}$, corresponding to $\alpha_{\mathrm{f}}=1 \%$ per $1 \mathrm{~Hz}$ subband.

This threshold is verified using $\mathrm{O} 2$ interferometric data. In three clean $1 \mathrm{~Hz}$ subbands, starting from $300 \mathrm{~Hz}, 600 \mathrm{~Hz}$, and $900 \mathrm{~Hz}, 200$ noise-only realizations are simulated by drawing random sky positions. The total 600 realizations yield a threshold $S_{\text {th }}=6.97$ ( $\alpha_{\mathrm{f}}=1 \%$ per $1 \mathrm{~Hz}$ subband), in good agreement with the threshold obtained in pure Gaussian noise. Hence we set $S_{\text {th }}=6.95$ in this search.

\section{Sensitivity}

We evaluate the search sensitivity by quantifying the signal strength $h_{0}$ required to achieve a 95\% detection efficiency, denoted by $h_{0}^{95 \%}$. It is first obtained by injecting 100 synthetic signals with a fixed $h_{0}$ value into Gaussian noise (ASD $S_{h}^{1 / 2}=4 \times 10^{-24} \mathrm{~Hz}^{-1 / 2}$ ) in four $1 \mathrm{~Hz}$ subbands, starting from $155 \mathrm{~Hz}, 355 \mathrm{~Hz}, 555 \mathrm{~Hz}$, and $755 \mathrm{~Hz}$. These simulations are marginalized over the orientation of the source, with the sky location fixed at the true position of Fomalhaut b. If more than $95 \%$ of the signals are correctly recovered, this process is repeated with a smaller value of $h_{0}$, and vice versa. We obtain an average of $h_{0}^{95 \%}=3.91 \times 10^{-26}$ in these four subbands.

This process is repeated in $\mathrm{O} 2$ interferometric data in a sample $1 \mathrm{~Hz}$ subband starting from $715 \mathrm{~Hz}$, randomly chosen from among the relatively clean bands. We obtain $h_{0}^{95 \%}=$ $1.67 \times 10^{-25}$, an order of magnitude larger than $h_{0}^{95 \%}$ obtained in Gaussian noise. This is because (1) the $\mathrm{O} 2$ noise ASD has not reached the design sensitivity of Advanced LIGO, and (2) the duty cycle (i.e., the proportion of time that the data are in analyzable science mode) of $\mathrm{O} 2$ is only about $50 \%$. The sensitivity in the full frequency band is presented in Sec. IV B and interpreted as the upper limits on $h_{0}$.

\section{RESULTS}

In this section, we describe the first-pass candidates obtained from the analysis with $S>S_{\text {th }}$ and a series of vetoes validating these candidates in Sec. IVA. After the veto procedure, only one candidate (with Viterbi score slightly above threshold) remains for further scrutiny. Without strong evidence of $\mathrm{CW}$, we present the strain upper limits and astrophysical interpretation in Sec. IV B.

\section{A. Candidates and vetoes}

We find in total 160 first-pass candidates with $S>S_{\text {th }}$. A large portion of the first-pass candidates are contributed 
TABLE II. Number of candidates remaining after each processing step. In total 160 first-pass candidates are found with $S>S_{\text {th }}=6.95$. After the five veto steps, one candidate remains for further scrutiny.

\begin{tabular}{lc}
\hline \hline Processing step & Candidates remaining \\
\hline First pass & 160 \\
Know-line veto & 96 \\
Single-interferometer veto & 13 \\
$T_{\text {obs }} / 2$ veto & 10 \\
Double $T_{\text {coh }}$ veto & 6 \\
Off-target veto & 1 \\
\hline \hline
\end{tabular}

from noise artifacts and the non-Gaussianity in the interferometric data. Narrow-band noise lines, e.g., $60 \mathrm{~Hz}$ power line harmonics, thermally excited mirror suspension violin modes, and lines from environmental disturbances are all sources of noise artifacts that obscure astrophysical CW signals. A five-step veto process is conducted to eliminate candidates resulting from noise artifacts [26]. See Table II for the number of candidates remaining after each step. See Fig. 2 for all the candidates with their Viterbi scores plotted as a function of frequency, as well as at which step each candidate is vetoed. The detailed veto criteria are described as follows.

First, we take into account the maximum Doppler shift due to the Earth's motion, $\delta f \approx 10^{-4} f_{0}$ (here $f_{0}$ is the starting frequency of the path), and widen the Viterbi path of each candidate by $\delta f$ on both sides of the path. We eliminate 64 candidates whose widened Viterbi paths intersect any known instrumental lines present in either the Hanford or Livingston interferometer [34,35].
Second, an additional 83 candidates are vetoed due to the contamination from not well understood artifacts in a single detector. The criteria are as follows. For each candidate, if searching data from a single interferometer yields $S \geq S_{\cup}$, where $S_{\cup}$ is the original score obtained with both interferometers combined, while searching the other interferometer yields $S<S_{\text {th }}$, and if the Viterbi path from the interferometer with $S \geq S_{\cup}$ intersects the original path, that candidate is vetoed. We eliminate 49 more candidates here. The above criterion is a stringent consistency check. After manually checking the remaining candidates, we find that a large portion of them are also caused by artifacts in a single detector, but do not necessarily meet the stringent criterion above. We inspect the candidate scores and paths manually and veto an additional 34 candidates due to contamination from a single detector. They fall into one of the three special cases: (a) We have $S>S_{\cup} \gg S_{\text {th }}$ in one detector and $S<S_{\text {th }}$ in the other; i.e., the candidate power completely comes from a single detector. The Viterbi path from the interferometer with $S \geq S_{\cup}$ does not intersect the original path (hence not automatically vetoed), but the two paths only differ by $\lesssim 0.02 \mathrm{~Hz}$. (b) We have a larger $S>S_{\cup} \gg S_{\text {th }}$ in one detector, but in the other we have $S$ slightly above $S_{\text {th }}$ (hence not automatically vetoed). The Viterbi path from the detector with $S>S_{\cup}$ intersects the original path, and the Viterbi path from the detector with $S \sim S_{\text {th }}$ does not intersect the original path. In addition, the higher $S$ from one detector is at least a number of 40 larger than $S$ from the other. (c) We have $S \sim S_{\cup} \gg S_{\text {th }}$ in one detector ( $S$ slightly below $S_{\cup}$, hence not automatically vetoed) and $S<S_{\text {th }}$ in the other. The Viterbi path from the detector with larger $S$ intersects the original path. In this

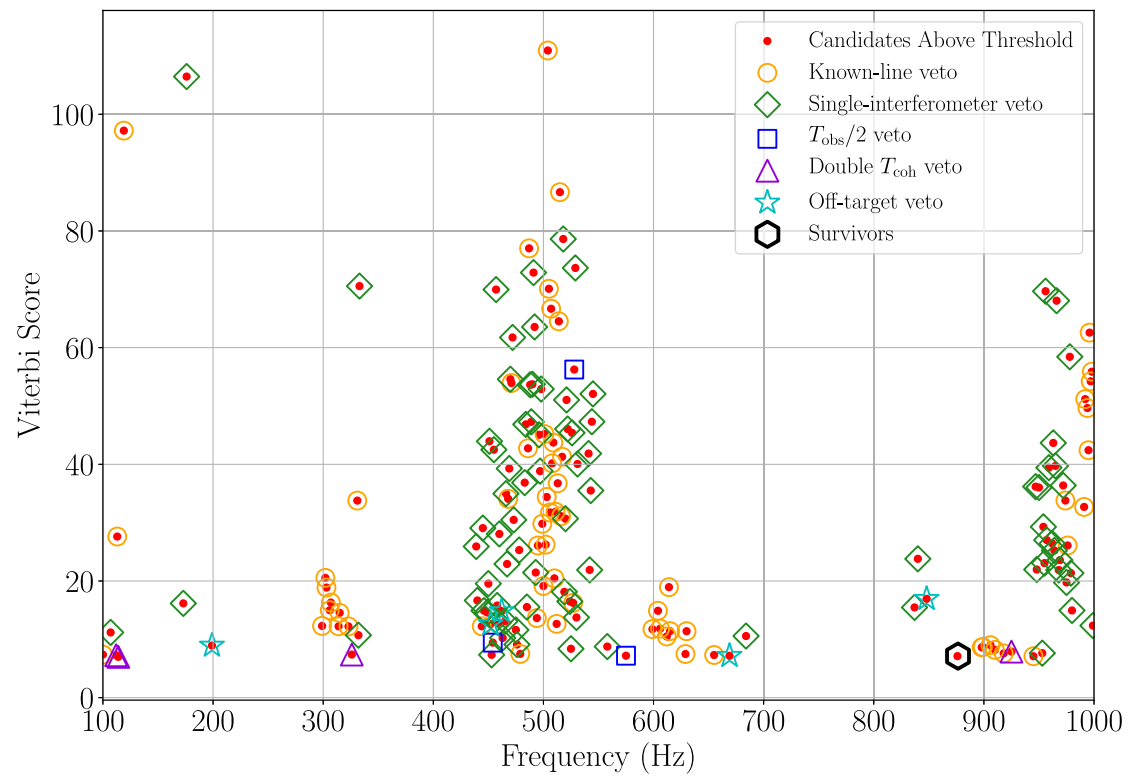

FIG. 2. Viterbi score $S$ of the first-pass candidate in each $1 \mathrm{~Hz}$ subband as a function of frequency (red dots). Candidates marked by orange circles, green diamonds, blue squares, purple triangles, and cyan stars are eliminated in stages 1-5 of the veto procedure (see Sec. IV A for details of the veto criteria). The only surviving candidate is marked by a black hexagon. 
case, the candidate is so loud that the Viterbi score is saturated around $S_{\cup} \gtrsim 50 \gg S_{\text {th }}$.

Third, an additional three candidates are vetoed by splitting the observation time into two halves and analyzing each half separately. A candidate is vetoed if the score obtained by searching one-half of $T_{\mathrm{obs}}$ is higher than or equal to $S_{\cup}$ (corresponding to the full $T_{\mathrm{obs}}$ ), while the score obtained in the other half is lower than $S_{\text {th }}$, and if the Viterbi path from the half with the higher score intersects the original path.

Fourth, the remaining ten candidates are further followed up by increasing $T_{\text {coh }}$. We increase $T_{\text {coh }}$ from five to ten days for a candidate whose estimated mean $\dot{f_{0}}$ over $T_{\text {obs }}$ is small enough such that Eq. (25) holds true when $T_{\text {coh }}=$ 10 d. Then, for each candidate, the $\mathcal{F}$-statistic and the Viterbi path are recomputed, using both interferometers combined. If the candidate is from astrophysical origin, the resulting Viterbi score with $T_{\text {coh }}=10 \mathrm{~d}$ should be higher than $S_{\cup}$, and the output Viterbi paths from searches with $T_{\text {coh }}=5 \mathrm{~d}$ and $10 \mathrm{~d}$ should match. Hence we veto a candidate if its score corresponding to $T_{\text {coh }}=10 \mathrm{~d}$ falls below $S_{\cup}$ and if the Viterbi paths from these two separate searches are at least $0.01 \mathrm{~Hz}$ apart. The candidate in the $462 \mathrm{~Hz}$ subband is excluded from this procedure because its estimated mean $\dot{f_{0}}$ is so large that $(25)$ is no longer satisfied if we increase $T_{\text {coh }}$ to $10 \mathrm{~d}$. We eliminate four candidates in this step.

Fifth, all but one candidate is eliminated using an "offtarget" veto. For each of the remaining six candidates, we first shift the sky location by increasing the right ascension by offsets ranging from $10 \mathrm{~s}$ to $1 \mathrm{hr}$ from the source's true location, with the declination fixed. We then increase the declination by offsets ranging from $10 \mathrm{~s}$ to $1 \mathrm{deg}$ from the source's true location, with the right ascension fixed at

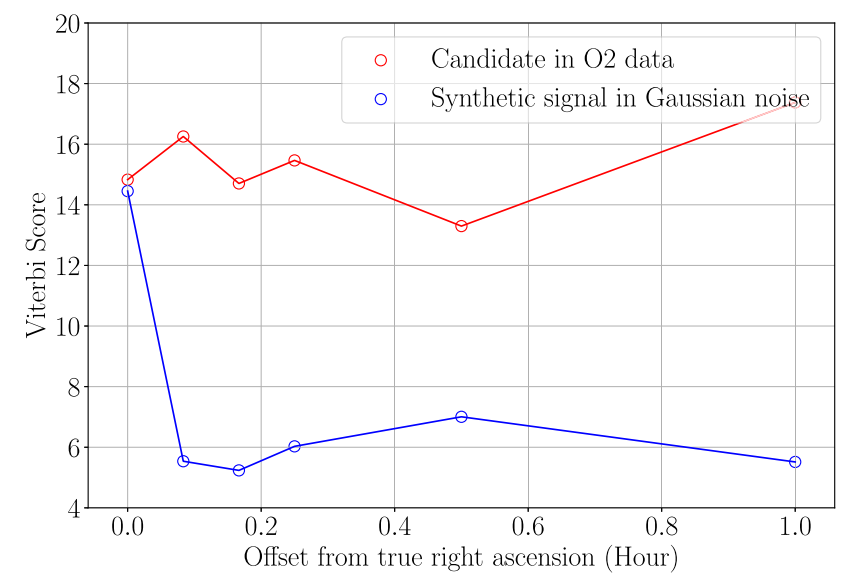

(a) the true value. We conduct the search and obtain the Viterbi score for each off-target position. A candidate is vetoed if this series of searches targeting sky locations shifted from the source's true location do not continuously yield Viterbi scores lower than the original score. That is, if the score does not drop below the threshold as we move away from the source's true location, we veto the candidate. The reliability of this veto is verified through synthetic signals injected into Gaussian noise. The same series of searches targeting the true sky location of the injection as well as the off-target locations is conducted. The simulation results demonstrate that the score drops below $S_{\text {th }}$ as we move away from the true sky location of the injection with an offset of $\sim 5^{\prime}$ in either right ascension or declination. A sample in the 462-463 Hz subband, which corresponds to a vetoed candidate in this step, is shown in Fig. 3.

The only candidate that survives the off-target veto is in the $876-877 \mathrm{~Hz}$ subband. The off-target search results in the real data in this subband behave exactly as those obtained from the simulations, as shown in Fig. 4. The Viterbi score immediately drops as we move away from the source's true location. The scores remain below threshold as we continue to increase the offset. Since this candidate behaves as an astrophysical signal, we keep it for further scrutiny.

Finally, the single remaining candidate in the $1 \mathrm{~Hz}$ subband starting at $876 \mathrm{~Hz}$ (path starts at $f_{0}=876.5034513780022 \mathrm{~Hz}$ ) is followed up using a technique in which the Doppler modulation, which accounts for the Doppler shift due to Earth's motion, is turned off when computing the $\mathcal{F}$-statistic [36], and the Viterbi score is recomputed in the same subband. A signal of astrophysical origin usually becomes undetectable and a different Viterbi path is returned when turning the Doppler

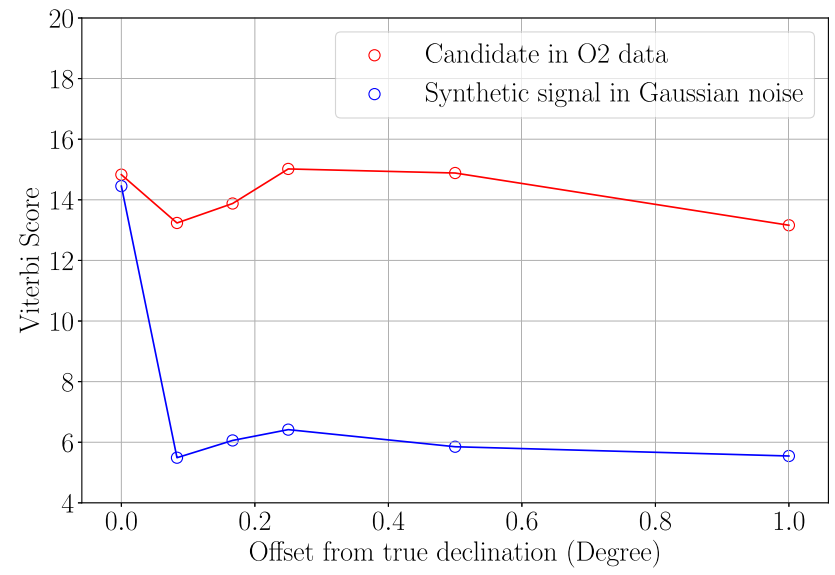

(b)

FIG. 3. Viterbi score as a function of the (a) right ascension and (b) declination offsets in the $1 \mathrm{~Hz}$ subband starting at $462 \mathrm{~Hz}$. The red and blue curves show the results obtained when following up the candidate in the real data and an injection in Gaussian noise, respectively. The results obtained for the candidate in real data show a steady and even increasing score as the offset increases. The simulation shows that the score drops below $S_{\text {th }}$ within $\sim 5^{\prime}$ from the true location (for either right ascension or declination). This candidate can therefore be vetoed. 


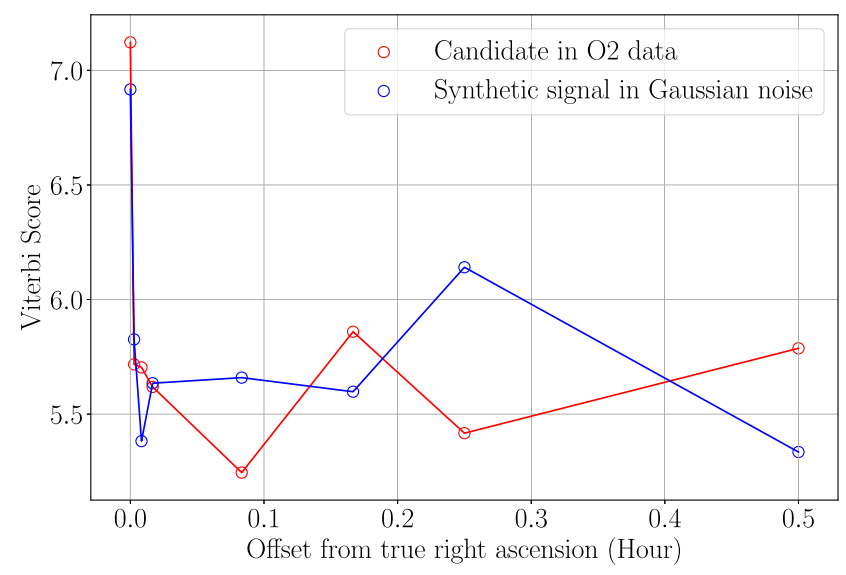

(a)

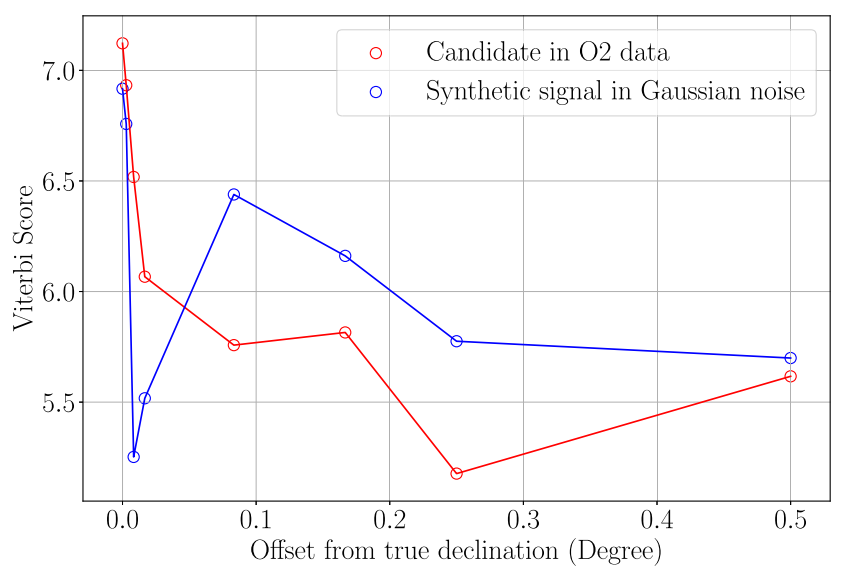

(b)

FIG. 4. Viterbi score as a function of the (a) right ascension and (b) declination offsets in the $1 \mathrm{~Hz}$ subband starting at $876 \mathrm{~Hz}$. The red and blue curves show the results obtained when following up the candidate in the real data and an injection in Gaussian noise, respectively. Both the scores obtained for the candidate in real data and the injection drop below threshold within $\sim 30^{\prime \prime}$ from the true location. The candidate survives this veto.

modulation off. However, a candidate caused by noise artifacts should yield a higher score and return a Viterbi path close to the original one; i.e., if the original Viterbi path is expanded by a frequency shift due to the Doppler modulation, the expanded path should intersect the new path returned when the Doppler modulation is turned off. Searching this final candidate with the Doppler modulation turned off yields a decreased Viterbi score 5.91 (cf. 7.12 in the original search). The newly returned Viterbi path differs from the original path by $\sim 0.005 \mathrm{~Hz}$ $\left(<10^{-4} f_{0}\right)$. The two paths are considered overlapped when taking into consideration the frequency shift due to the Earth's orbit. The results for this candidate do not confidently show consistency with either an astrophysical signal or an outlier caused by noise artifacts. Therefore, while the candidate cannot be vetoed at this point, the possibility that it comes from astrophysical origin is low. Given the threshold chosen for $\alpha_{\mathrm{f}}=1 \%$ per subband and the fact that the original score of the candidate is only slightly above this threshold $\left(S-S_{\text {th }}=0.17\right)$, the final candidate in subband $876-877 \mathrm{~Hz}$ could possibly be a false alarm. We provide the full Viterbi path of the final candidate in Appendix and recommend following it up in future observing runs, including the data already collected in the third observing run.

\section{B. Strain upper limits}

No strong evidence of continuous waves is found in this search. We place the upper limits on the signal strain at $90 \%$ confidence level, denoted by $h_{0}^{90 \%}$, as a function of frequency. Figure 5 shows $h_{0}^{90 \%}$ in all 741 subbands where no candidate is vetoed (blue dots), as well as 22 sample $h_{0}^{95 \%}$ values obtained from injections (orange stars). The procedure of producing these results is as follows. First, we derive the upper limits at $95 \%$ confidence level empirically in 22 randomly selected sample subbands, marked by the orange stars. A set of 100 synthetic signals are injected into the $\mathrm{O} 2$ data at the sky position of Fomalhaut $\mathrm{b}$ in each subband with a fixed $h_{0}$. The other source parameters are randomly drawn from their uniform distributions. The corresponding detection rate is calculated. This process is repeated with different $h_{0}$ values with step size $1 \times 10^{-27}$ until $95 \%$ of the injections are recovered. Next, we calculate the sensitivity depth $\mathcal{D}=\sqrt{S_{h}(f)} / h_{0}^{95 \%}(f)$ in these 22 sample subbands. Here $\sqrt{S_{h}(f)}$ is the effective ASD calculated from the harmonic mean of the two detectors over all the 30 min SFTs collected from GPS time 1180310418-1187733592 ( 3 months). In order to save computing cost, we use the minimum depth in the 22 sample subbands, $\mathcal{D}_{\min }$, as the property of the search pipeline and calculate the strain upper limits in the full band, i.e., $\sqrt{S_{h}(f)} / \mathcal{D}_{\text {min }}$ (the blue dots). The subbands containing vetoed candidates, where no upper limit can be placed, are excluded. Finally, we correct for the confidence level of these upper limits calculated in the full band. We denote the ratio of all the subbands satisfying $\mathcal{D} \geq \mathcal{D}_{\min }$ to the total 741 subbands by $\beta$. By construction, we have $\mathcal{D} \geq \mathcal{D}_{\text {min }}$ in all 22 randomly chosen subbands. The probability for this to happen in any 22 randomly chosen subbands can be approximated by $\beta^{22}$ (when the number of sample subbands is much smaller than the total number of subbands). By assuming that this probability is at least $95 \%$, we get $\beta \geq 0.95^{1 / 22}$. In any randomly selected subband, there is at least $95 \%$ chance that the signal can be detected if $\mathcal{D} \geq \mathcal{D}_{\text {min }}$; the probability of having $\mathcal{D} \geq \mathcal{D}_{\text {min }}$ in that subband is $\beta$; and the probability for $\beta \geq 0.95^{1 / 22}$ to be valid is at least $95 \%$. Hence the new confidence level in the full band is at least $90 \%$ 


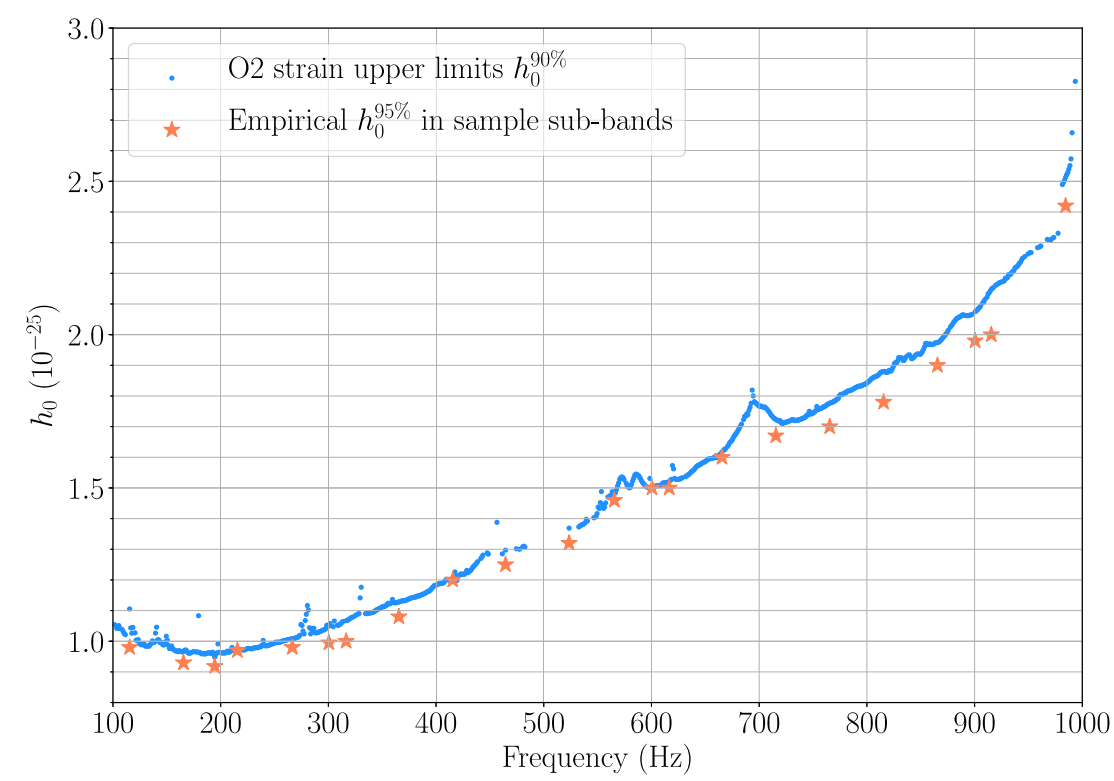

FIG. 5. The strain upper limits as a function of frequency. The blue dots represent the $90 \%$ confidence level strain upper limits, $h_{0}^{90 \%}$, in the full band, and the orange stars represent the 22 sample $1 \mathrm{~Hz}$ subbands where the $h_{0}^{95 \%}$ values were obtained empirically. The subbands without a marker were vetoed $[42,43]$.

(i.e., $\left.0.95 \times 0.95^{1 / 22} \times 0.95\right)$. We denote the strain upper limits in the full band by $h_{0}^{90 \%}$. In any given subband, there is a probability lower than $10 \%$ that a signal with $h_{0} \geq h_{0}^{90 \%}$ is missed in this search.

The indirect upper limit on signal strain due to energy conservation, inferred from the age and distance of the source, is given by $[13,37]$

$$
h_{0}^{\mathrm{age}}=1.26 \times 10^{-24}\left(\frac{3.3 \mathrm{kpc}}{d}\right)\left(\frac{300 \mathrm{yr}}{t_{\mathrm{age}}}\right)^{1 / 2},
$$

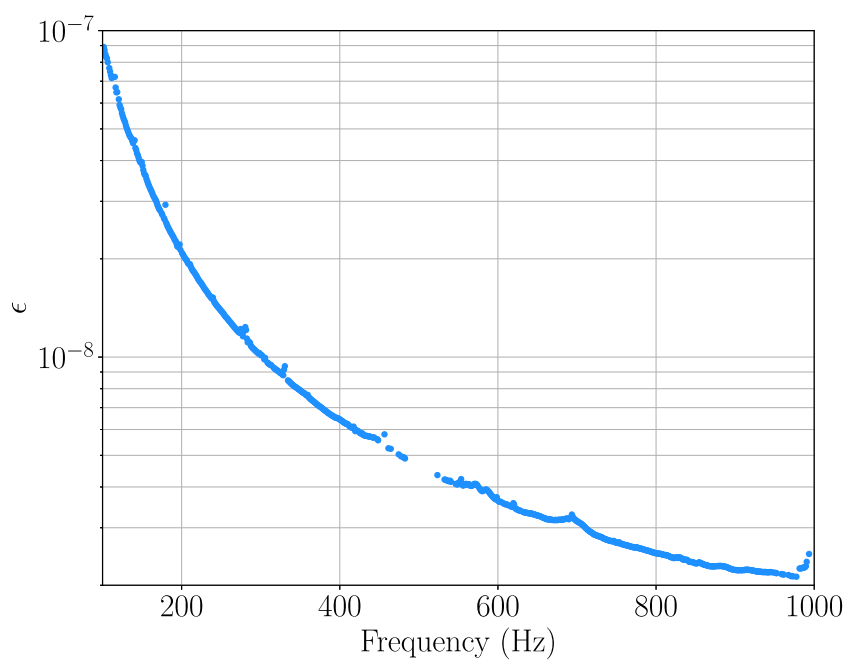

(a) where $d$ is the distance to the star. Assuming that Fomalhaut $\mathrm{b}$ is a neutron star with $t_{\mathrm{age}}=3000 \mathrm{kyr}$ and $d=0.02 \mathrm{kpc}$ (the pessimistic scenario presented in Ref. [13]), we have $h_{0}^{\text {age }}=2.08 \times 10^{-24}$. The upper limits obtained in this analysis beat the indirect limit $h_{0}^{\text {age }}$ in the full band searched. We compare the strain upper limits obtained from this search to the previous results in Ref. [13]. In the most sensitive band around $200 \mathrm{~Hz}$, $h_{0}^{95 \%}=1.2 \times 10^{-25}$ is obtained in Advanced LIGO's first observing run, as presented in Ref. [13]. In this search, the strain upper limit in the most sensitive $1 \mathrm{~Hz}$ subband

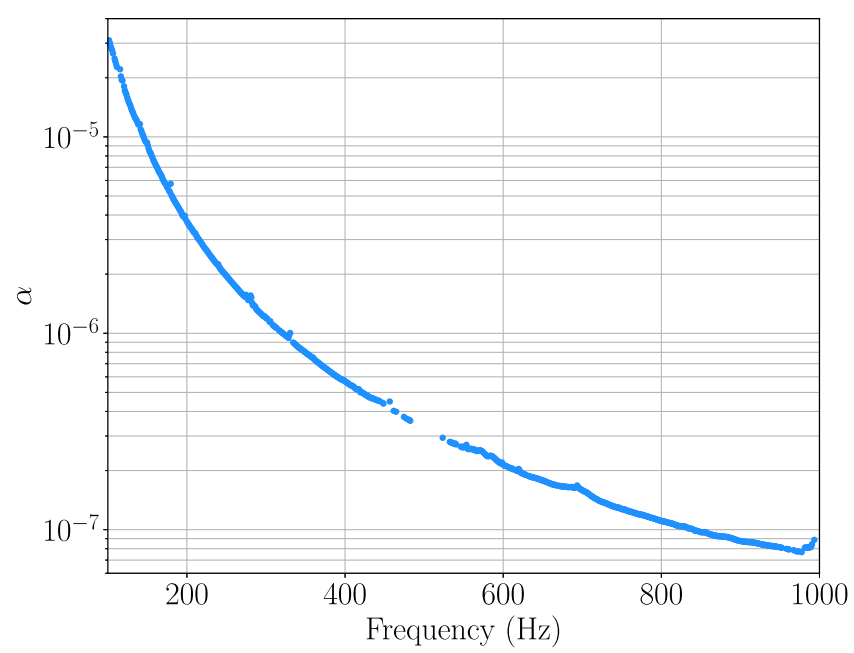

(b)

FIG. 6. Upper limits on (a) fiducial ellipticity $\epsilon$ and (b) $r$-mode amplitude $\alpha$, converted from the strain upper limits in Fig. 5 $($ see $[42,43])$. 
starting from $194 \mathrm{~Hz}, h_{0}^{90 \%}=9.48 \times 10^{-26}$, improves on previously published results by a factor of 1.3 . We also compare our results to the $h_{0}$ upper limits reported in all-sky searches for CWs using the $\mathrm{O} 2$ data. Our most constraining $h_{0}^{90 \%}$ is a factor of 1.8 and 1.4 lower than the best upper limits $h_{0}^{95 \%} \simeq 1.7 \times 10^{-25}$ at around $120 \mathrm{~Hz}$ reported in Ref. [18], and $h_{0}^{90 \%}=1.3 \times 10^{-25}$ at $163 \mathrm{~Hz}$ reported in Ref. [38], respectively. At higher frequencies in the range of 500-1000 Hz, our upper limits $\sim 1.3 \times 10^{-25}-2.7 \times 10^{-25}$ are comparable and slightly better than those $\sim 1.5 \times 10^{-25}-3 \times 10^{-25}$ reported in an all-sky search targeting low-ellipticity sources [39].

We then convert $h_{0}^{90 \%}$ to the upper limits on the fiducial ellipticity of the neutron star [13,37]

$$
\epsilon=9.5 \times 10^{-5}\left(\frac{h_{0}}{1.2 \times 10^{-24}}\right)\left(\frac{d}{1 \mathrm{kpc}}\right)\left(\frac{100 \mathrm{~Hz}}{f}\right)^{2},
$$

and the $r$-mode amplitude parameter $[13,40,41]$

$$
\alpha=0.028\left(\frac{h_{0}}{10^{-24}}\right)\left(\frac{d}{1 \mathrm{kpc}}\right)\left(\frac{100 \mathrm{~Hz}}{f}\right)^{3} .
$$

Figure 6 shows the upper limits on $\epsilon$ and $\alpha$ as a function of frequency, assuming a distance of $d=0.011 \mathrm{kpc}$, and the principal moment of inertia $I_{z z}=10^{45} \mathrm{~g} \mathrm{~cm}^{2}$.

\section{CONCLUSION}

We report the results from a directed search for $\mathrm{CW}$ signals from the neutron star candidate Fomalhaut $\mathrm{b}$ in the frequency band 100-1000 Hz, using Advanced LIGO O2 data. An efficient, semicoherent search method, based on a HMM tracking scheme and a matched filter $\mathcal{F}$-statistic, is used to track the signal frequency. After passing the abovethreshold candidates through five veto steps, only one candidate survives. The final surviving candidate appears to be consistent with the false alarm probability, and no compelling evidence of continuous waves is found. Upper limits on $h_{0}$ with $90 \%$ confidence are calculated in the full frequency band. In the most sensitive subband, 194-195 Hz, we obtain $h_{0}^{90 \%}=9.48 \times 10^{-26}$.

In the future, the search may benefit from new information gathered from electromagnetic observations of the source, as well as the further improvements to the instruments. In particular, the final candidate in the subband $876-877 \mathrm{~Hz}$ can be followed up in the third observing run or future runs of Advanced LIGO and Virgo. Regardless of the eventual outcome of this search, this methodology represents an important development in the search for CWs and could easily be extended to other isolated neutron star sources.

\section{ACKNOWLEDGMENTS}

This research has made use of data, software, and/or web tools obtained from the Gravitational Wave Open Science Center [42], a service of LIGO Laboratory, the LIGO Scientific Collaboration and the Virgo Collaboration. LIGO is funded by the U.S. National Science Foundation. Virgo is funded by the French Centre National de Recherche Scientifique (CNRS), the Italian Istituto Nazionale della Fisica Nucleare (INFN), and the Dutch Nikhef, with contributions by Polish and Hungarian institutes. The authors thank the LIGO Laboratory for providing the resources with which to conduct this search, as well as Alan Weinstein and the LIGO SURF program, the National Science Foundation, and the California Institute of Technology for sponsoring the project. The authors also thank Meg Millhouse for the review and suggestions. L. S. is a member of the LIGO Laboratory. LIGO was constructed by the California Institute of Technology and Massachusetts Institute of Technology with funding from the United States National Science Foundation, and operates under cooperative agreement PHY-1764464. Advanced LIGO was built under Grant No. PHY-0823459. L. S. also acknowledges the support of the Australian Research Council Centre of Excellence for Gravitational Wave Discovery (OzGrav), Project No. CE170100004. The authors thank all of the essential workers who put their health at risk during the COVID-19 pandemic, without whom we would not have been able to complete this work. This paper carries LIGO Document No. LIGO-P2000118. 


\section{APPENDIX: VITERBI PATH OF THE CANDIDATE IN 876-877 HZ BAND}

Here we provide the output Viterbi path for the final candidate surviving all vetoes in Sec. IVA. The frequencies at discrete time steps are listed in Table III and plotted in Fig. 7.

TABLE III. Frequencies at each step on the candidate Viterbi path.

\begin{tabular}{|c|c|}
\hline Step & Frequency $[\mathrm{Hz}]$ \\
\hline 1 & 876.5034513780022 \\
\hline 2 & 876.5034513780022 \\
\hline 3 & 876.5034513780022 \\
\hline 4 & 876.5034502205948 \\
\hline 5 & 876.5034490631874 \\
\hline 6 & 876.5034490631874 \\
\hline 7 & 876.5034479057800 \\
\hline 8 & 876.5034479057800 \\
\hline 9 & 876.5034479057800 \\
\hline 10 & 876.5034479057800 \\
\hline 11 & 876.5034479057800 \\
\hline 12 & 876.5034467483727 \\
\hline 13 & 876.5034455909653 \\
\hline 14 & 876.5034455909653 \\
\hline 15 & 876.5034444335579 \\
\hline 16 & 876.5034432761505 \\
\hline 17 & 876.5034432761505 \\
\hline 18 & 876.5034421187431 \\
\hline 19 & 876.5034421187431 \\
\hline 20 & 876.5034409613357 \\
\hline 21 & 876.5034398039284 \\
\hline 22 & 876.5034386465210 \\
\hline 23 & 876.5034374891136 \\
\hline 24 & 876.5034363317062 \\
\hline 25 & 876.5034351742988 \\
\hline 26 & 876.5034340168914 \\
\hline 27 & 876.5034328594841 \\
\hline 28 & 876.5034317020767 \\
\hline 29 & 876.5034317020767 \\
\hline 30 & 876.5034305446693 \\
\hline 31 & 876.5034305446693 \\
\hline 32 & 876.5034305446693 \\
\hline 33 & 876.5034293872619 \\
\hline 34 & 876.5034293872619 \\
\hline 35 & 876.5034293872619 \\
\hline 36 & 876.5034293872619 \\
\hline 37 & 876.5034293872619 \\
\hline 38 & 876.5034293872619 \\
\hline 39 & 876.5034293872619 \\
\hline 40 & 876.5034282298545 \\
\hline 41 & 876.5034270724472 \\
\hline 42 & 876.5034259150398 \\
\hline 43 & 876.5034247576324 \\
\hline 44 & 876.5034236002250 \\
\hline 45 & 876.5034224428176 \\
\hline 46 & 876.5034212854102 \\
\hline 47 & 876.5034212854102 \\
\hline
\end{tabular}




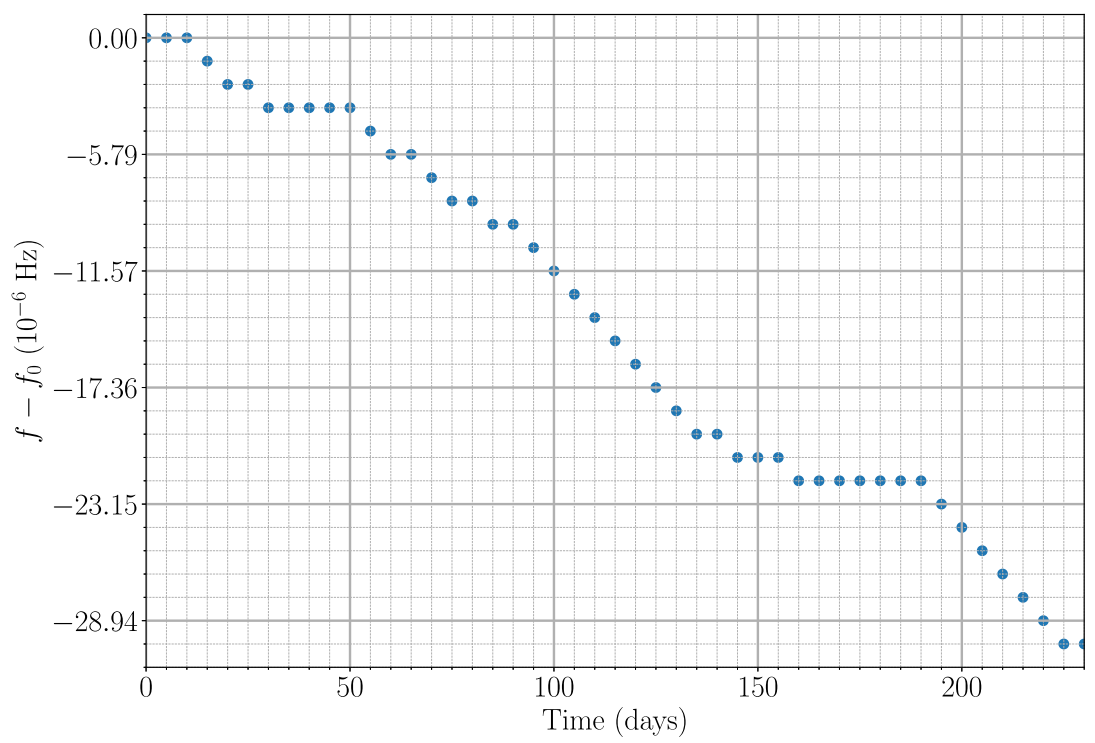

FIG. 7. Viterbi path of the final candidate surviving all vetoes in the subband $876-877 \mathrm{~Hz}$. The starting frequency equals the frequency at the first step in Table III, $f_{0}=876.5034513780022 \mathrm{~Hz}$.

[1] B. P. Abbott et al. (LIGO Scientific and Virgo Collaborations), Observation of Gravitational Waves from a Binary Black Hole Merger, Phys. Rev. Lett. 116, 061102 (2016).

[2] J. Aasi et al. (LSC Collaboration), Advanced LIGO, Classical Quantum Gravity 32, 074001 (2015).

[3] F. Acernese et al. (Virgo Collaboration), Advanced Virgo: A second-generation interferometric gravitational wave detector, Classical Quantum Gravity 32, 024001 (2015).

[4] B. P. Abbott et al. (LIGO Scientific and Virgo Collaborations), GWTC-1: A Gravitational-Wave Transient Catalog of Compact Binary Mergers Observed by LIGO and Virgo during the First and Second Observing Runs, Phys. Rev. X 9, 031040 (2019).

[5] B. P. Abbott et al., GW190412: Observation of a binaryblack-hole coalescence with asymmetric masses, Phys. Rev. D 102, 043015 (2020).

[6] B. P. Abbott et al., GW190425: Observation of a compact binary coalescence with total mass $\sim 3.4 \mathrm{~m} \odot$, Astrophys. J. 892, L3 (2020).

[7] R. Abbott et al., GW190814: Gravitational waves from the coalescence of a 23 solar mass black hole with a 2.6 solar mass compact object, Astrophys. J. Lett. 896, L44 (2020).

[8] K. Riles, Recent searches for continuous gravitational waves, Mod. Phys. Lett. A 32, 1730035 (2017).

[9] B. P. Abbott et al. (LIGO Scientific and Virgo Collaborations), Narrow-band search for gravitational waves from known pulsars using the second LIGO observing run, Phys. Rev. D 99, 122002 (2019).

[10] B. P. Abbott et al., Searches for gravitational waves from known pulsars at two harmonics in 2015-2017 LIGO data, Astrophys. J. 879, 10 (2019).
[11] B. P. Abbott et al., Upper limits on gravitational waves from Scorpius X-1 from a model-based cross-correlation search in Advanced LIGO data, Astrophys. J. 847, 47 (2017).

[12] B. P. Abbott et al. (LIGO Scientific and Virgo Collaborations), Search for gravitational waves from Scorpius X-1 in the second Advanced LIGO observing run with an improved hidden Markov model, Phys. Rev. D 100, 122002 (2019).

[13] B. P. Abbott et al., Searches for continuous gravitational waves from 15 supernova remnants and Fomalhaut b with Advanced LIGO, Astrophys. J. 875, 122 (2019).

[14] L. Lindblom and B. J. Owen, Directed searches for continuous gravitational waves from twelve supernova remnants in data from Advanced Ligo's second observing run, Phys. Rev. D 101, 083023 (2020).

[15] M. Millhouse, L. Strang, and A. Melatos, Search for gravitational waves from 12 young supernova remnants with a hidden Markov model in Advanced LIGO's second observing run, Phys. Rev. D 102, 083025 (2020).

[16] J. Ming, M. A. Papa, A. Singh, H.-B. Eggenstein, S. J. Zhu, V. Dergachev, Y. Hu, R. Prix, B. Machenschalk, C. Beer, O. Behnke, and B. Allen, Results from an Einstein@Home search for continuous gravitational waves from Cassiopeia A, Vela Jr., and G347.3, Phys. Rev. D 100, 024063 (2019).

[17] L. Fesik and M. A. Papa, first search for r-mode gravitational waves from PSR J0537-6910, Astrophys. J. 895, 11 (2020).

[18] B. P. Abbott et al. (LIGO Scientific and Virgo Collaborations), All-sky search for continuous gravitational waves from isolated neutron stars using Advanced LIGO O2 data, Phys. Rev. D 100, 024004 (2019). 
[19] R. Abbott et al., Open data from the first and second observing runs of Advanced LIGO and Advanced Virgo, arXiv:1912.11716.

[20] T. Currie, J. Debes, T. J. Rodigas, A. Burrows, Y. Itoh, M. Fukagawa, S. J. Kenyon, M. Kuchner, and S. Matsumura, Direct imaging confirmation and characterization of a dustenshrouded candidate exoplanet orbiting Fomalhaut, Astrophys. J. 760, L32 (2012).

[21] P. Kalas, J. R. Graham, E. Chiang, M. P. Fitzgerald, M. Clampin, E. S. Kite, K. Stapelfeldt, C. Marois, and J. Krist, Optical images of an exosolar planet 25 light-years from earth, Science 322, 1345 (2008).

[22] R. Neuhuser, M. M. Hohle, C. Ginski, J. G. Schmidt, V. V. Hambaryan, and T. O. B. Schmidt, The companion candidate near Fomalhaut a background neutron star?, Mon. Not. R. Astron. Soc. 448, 376 (2015).

[23] A. Gáspár and G. H. Rieke, New HST data and modeling reveal a massive planetesimal collision around Fomalhaut, Proc. Natl. Acad. Sci. U.S.A. 117, 9712 (2020).

[24] G. Hobbs, A. G. Lyne, and M. Kramer, An analysis of the timing irregularities for 366 pulsars, Mon. Not. R. Astron. Soc. 402, 1027 (2010).

[25] L. Sun, A. Melatos, S. Suvorova, W. Moran, and R. J. Evans, Hidden markov model tracking of continuous gravitational waves from young supernova remnants, Phys. Rev. D 97, 043013 (2018).

[26] B. P. Abbott et al. (LIGO Scientific and Virgo Collaborations), Search for gravitational waves from Scorpius X-1 in the first Advanced LIGO observing run with a hidden Markov model, Phys. Rev. D 95, 122003 (2017).

[27] L. Sun and A. Melatos, Application of hidden Markov model tracking to the search for long-duration transient gravitational waves from the remnant of the binary neutron star merger GW170817, Phys. Rev. D 99, 123003 (2019).

[28] L. Sun, R. Brito, and M. Isi, Search for ultralight bosons in Cygnus X-1 with Advanced Ligo, Phys. Rev. D 101, 063020 (2020).

[29] L. Sun, A. Melatos, and P. D. Lasky, Tracking continuous gravitational waves from a neutron star at once and twice the spin frequency with a hidden Markov model, Phys. Rev. D 99, 123010 (2019).

[30] P. Jaranowski, A. Królak, and B. F. Schutz, Data analysis of gravitational-wave signals from spinning neutron stars:
The signal and its detection, Phys. Rev. D 58, 063001 (1998).

[31] R. Prix, Search for continuous gravitational waves: Metric of the multidetector F-statistic, Phys. Rev. D 75, 023004 (2007).

[32] C. Cutler and B. F. Schutz, Generalized $\mathcal{F}$-statistic: Multiple detectors and multiple gravitational wave pulsars, Phys. Rev. D 72, 063006 (2005).

[33] A. Viterbi, Error bounds for convolutional codes and an asymptotically optimum decoding algorithm, IEEE Trans. Inf. Theory 13, 260 (1967).

[34] P. B. Covas et al. (LSC Instrument Authors), Identification and mitigation of narrow spectral artifacts that degrade searches for persistent gravitational waves in the first two observing runs of advanced ligo, Phys. Rev. D 97, 082002 (2018).

[35] Gravitational Wave Open Science Center-O2 instrumental lines, https://www.gw-openscience.org/o2speclines/.

[36] S. J. Zhu, M. A. Papa, and S. Walsh, New veto for continuous gravitational wave searches, Phys. Rev. D 96, 124007 (2017).

[37] K. Wette et al., Searching for gravitational waves from cassiopeia a with LIGO, Classical Quantum Gravity 25, 235011 (2008).

[38] B. Steltner, M. A. Papa, H.-B. Eggenstein, B. Allen, V. Dergachev, R. Prix, B. Machenschalk, S. Walsh, S. J. Zhu, and S. Kwang, Einstein@ Home all-sky search for continuous gravitational waves in LIGO O2 public data, arXiv:2009 .12260 .

[39] V. Dergachev and M.A. Papa, Results from the First All-Sky Search for Continuous Gravitational Waves from Small-Ellipticity Sources, Phys. Rev. Lett. 125, 171101 (2020).

[40] B. J. Owen, How to adapt broad-band gravitational-wave searches for $r$-modes, Phys. Rev. D 82, 104002 (2010).

[41] L. Lindblom, B. J. Owen, and S. M. Morsink, Gravitational Radiation Instability in Hot Young Neutron Stars, Phys. Rev. Lett. 80, 4843 (1998).

[42] http://link.aps.org/supplemental/10.1103/PhysRevD.103 .023020

[43] See Supplemental Material at http://link.aps.org/ supplemental/10.1103/PhysRevD.103.023020 for the source data in Figs. 5 and 6. 